\title{
Skin Tumors Diagnosis Utilizing Case Based Reasoning and The Expert System
}

\author{
Roza Fuad Majeed \\ Information Technology Department \\ Technical College of Informatics \\ Sulaimani Polytechnic University \\ Sulaimani, Iraq \\ Roza.fuad@spu.edu.iq
}

Dana Abdulmajeed Abdilkarim

Medical laboratory

Technical College of Health

Sulaimani Polytechnic University

Sulaimani, Iraq

Dana77@doctor.com

\author{
Soran AB. M. Saeed \\ Vice presidence of \\ Scientific Affairs \\ Sulaimani Polytechnic University \\ Sulaimani, Iraq \\ $\underline{\text { Soran.saeed@ spu.edu.iq }}$
}

\author{
Haval Mohammed Sidqi \\ Database Department \\ Computer Science Institute \\ Sulaimani Polytechnic University \\ Sulaimani, Iraq \\ Haval.Sidqi@spu.edu.iq
}

\begin{tabular}{l}
\hline Article Info \\
\hline Volume 5 - Issue 1 - \\
June 2020 \\
DOI: \\
10.24017/science.2020.1.10 \\
Article history: \\
Received: 26 January 2020 \\
Accepted: 12 March 2020 \\
\hline
\end{tabular}

\section{Keywords:}

Skin cancer,

$\mathrm{BCC}$,

$\mathrm{SCC}$,

melanoma,

CBR,

expert system.

\section{ABSTRACT}

Skin cancer is considered as the most type of cancer that happens in humans. Three basic types of cancer occur which are basal cell carcinoma (BCC), Squamous cell carcinoma (SCC). Skin cancer leads to death if it is not diagnosed in an early stage. Fortunately, early diagnosis of skin cancer raises the survival rate of victims. Computer-aided has a great role to detect skin cancer which leads to saving human life. Based on that, this study proposes a computer-aided diagnosis (CAD) system that detects skin cancer using digital images, techniques of image processing, by using the case-based reasoning and expert system. The main goal for designing this system is to create a cheap, easy-to-use, and relatively accurate system for detecting skin cancer in an early stage to save human life, raises the survival rate, and decreases the cost of the dermoscopy test. 


\section{INTRODUCTION}

Skin is the largest part of the human body; it protects our body from heat and cold. The skin also protects the body from Physical hazards such as Ultraviolet light, Mechanical hazards such as traumas and friction, biological hazards such as viruses, bacteria and fungi. The skin consists of three layers which are epidermis, dermis and the subcutaneous adipose tissue. Fifteen percentage weight of the body is the skin [1].Cancer is considered as a common issue of clinical medicine. It is known by the abnormal growth of the cells which leads to death if it is not controlled. The unusual growth of the cell that remains in the same location is called benign, while the abnormal cells that do not remain in the same location or the cells spread then it is called malignant. Malignant tumors divided into two types, a primary tumor and secondary tumor. Skin cancer is a type of cancer and considered as the common type of cancers which is approximately \%50 of all cancer is skin cancer. Skin cancer happens in peoples who have fair- skin and in the people exposed to the sun and ultraviolet light for a long time and in the people who are exposed to the $\mathrm{x}$ - radiation [2]. Skin cancer divided into three types two of them are non- melanoma skin cancer (NMSC) and they are basal cell carcinoma which is the common type, squamous cell, the third type of skin cancer is melanoma which is known as melanoma skin cancer (MSC) [3]. Computer-aided detection or diagnosis (CAD) systems have a great role in the field of Medical Image Diagnosis. It helps dermatologists to raise the accuracy. For classifying image of skin into benign and malignant different rules can be used such as image processing techniques by using pre-processing and extracting feature parameters [4], [5].

In this paper, case-based reasoning used which is a branch of artificial intelligence (AI), an expert system for the implementation of the proposed system image processing techniques are used in the pre-processing step for analyzing the image, and a database used for the implementation. Over 110 images collected as real cases from Emergency hospitals and from the Dermatology hospital and from the Ali Kamal hospital and also from the private clinics. The aim of this paper is to present the techniques and analyze the image of the skin lesion and design the proposed system and to implement the system for detecting skin cancer in an early stage in order to save human life and decreasing cost for recognizing skin cancer.

\section{LITERATURE REVIEW}

In recent days, skin cancer is the most common and most hazardous type of cancer found in humans. Computer vision plays an important role in Medical Image Diagnosis. In this field, there are many authors using different rules and methods for skin cancer detection and classify the image of the skin as a benign or malignant.

In a related study conducted by Fernandez et al. [6] proposed an intelligent system and they used the following methods for running the system. In the beginning they used the image that was taken from the International Skin Imaging Collaboration (ISIC), after that the image segmentation started by applying the Wavelet - Fuzzy C-Means algorithm. Next, the feature extraction stage begins. Authors determined the specific features, and also determined the regions-of-interest (ROI), then they used ABCD guide for extracting the feature, and an ensemble of clusters that were able to extract the Red-Green-Blue values. The extraction feature stage includes the Grey Level Co-Occurrence Matrix (GLCM) method. Finally, the proposed system made a classification for the image into a benign or malignant tumor, they used ROC curve for calculating accuracy, specificity, sensibility. Another two authors worked on melanoma skin cancer detection in Periyasamy et al. [7] the MR image of the human skin was used, and convert the images into a binary image, in addition, pre-processing done on the images that consist of two parts, the first part is resizing the image to the perfect size for the detection system. The second part is hair removal by using the Frangi vesselness algorithm. For image segmentation, the K-Means clustering algorithm was used. Features extracted from the image by using the ABCD rule, and classify the images into normal skin or malignant. In the Anas et al. [8] proposed a system classification for skin cancer, they used a combination of the color and texture features, because this combination gave the best result compared to using 
gray level information or color alone. They made segmentation for the image of the lesions by using the k-means clustering algorithm, feature extraction made by six different color-textures from the segmented image. The accuracy of the proposed system evaluated on four types of classifier, and the value of the system compared with one another, and to perform better analysis the result of the evaluation of the proposed system compared with five classification rates. Khirala et al. [9] wavelet function introduced to the interested technical person outside of the digital signal processing field. The history of the wavelets described that the wavelets beginning with Fourier, and comparison done between the wavelet transform and the Fourier transforms, and they discussed their properties and other special aspects of wavelets and also include some interesting applications such as de-noising noisy data, musical tones, and image compression. Kolkur et al. [10] discussed various classifiers for skin disease detection. Authors collected data for ten common skin lesions; they applied different classifiers on the skin lesions such as K Nearest Neighbors (KNN), Support Vector Machine (SVM), Decision Tree, and Artificial Neural Networks (ANN), after applying the classifier the result showed that the classifier that has the best Accuracy and performance is the Artificial Neural Networks (ANN). Moreover, Chandrahasa et al. [5] made a Smartphone application for skin cancer (melanoma) detection in early stage, they used the (ABCDE) properties which means Asymmetry, Border, Color variation, Diameter and Expansion for the lesion. They used many different techniques for image processing such as converting the RGB color image into the grayscale image and segmentation for the image and contour and histogram analysis. Shukran et al. [11] made diagnosing for melanoma skin cancer by using image processing techniques the process begins with input the image of the skin lesion the dermoscopy image and then the image pre-processed by using techniques for resizing image and de-noise for the image such as hair removal after that the denoised image go to the another stage which is segmentation for the image by using clustering and thresholding and another step is feature extraction by using the $(\mathrm{ABCDE})$ rule and the image classified in to the normal or cancer. This article also presented the possible methods for detection steps and provided some of the possible classifiers for classification of the image such as ANN classifier and artificial intelligence methodologies. Bindhu et al. [12] proposed a system for the segmentation of skin lesions by using MATLAB for implementing the proposed system. The image used in the system captured by the digital camera for locating the skin lesion for the digital image they used Texture Distinctiveness Lesion Segmentation (TDLS) for the segmentation of the image and find the regions-of-interest (ROI). In the TDLS method, the method learned the texture of the skin and the lesion then made a calculation for the TD metric and classifies the image as a part of the lesion class or part of the skin class. Annan et al. [13] made a review on the image segmentation methods and techniques that are widely used in the field of image segmentation. They reviewed the following algorithms such as Segmentation by Edge Detection, Segmentation by Region-based, Segmentation by Thresholding, and Segmentation by Featurebased Clustering. The images analysis's outcome gave the result that the thresholding and region growing gives better results than edge detection and clustering. Lamy et al. [14] made an article for using case-based reasoning in the breast cancer field. They proposed the CBR method that automatically executed and presented visually, the system combined qualitative and quantitative approaches. The proposed method tested on three public datasets that have classification accuracy comparable to the k-Nearest Neighbor (KNN) algorithm. Through the small user study, they tested the proposed method for the better explainable. Kawahara et al. [15]demonstrated a linear classifier that classified 10 varies skin lesions, feature extracted by using the modified Alex Net from the fully connected layer to the Convolutional layer this modified features applied on 1300 clinical images of 10 class dataset of skin lesions with the accuracy of $81.8 \%$. Vijayalakshmi [16] Presented a fully automated system for recognizing the dermatological disease through image of the skin lesion. The system consists of three phases which are compromising data collection and augmentation, designing model and prediction. Multiple AI algorithms used such as, Support Vector Machine and Convolutional Neural Network and amalgamated it with image processing tools the system has with the accuracy of $85 \%$. Codella et al. [17] Presents an approach for recognizing melanoma in 
dermoscopy images that combines sparse coding, deep learning, and Support vector machine (SVM) learning algorithms. Unsupervised learning used within the domain and feature transferred from the domain of natural photographs. Methodology and performance evaluated on a dataset from the International Skin Imaging Collaboration (ISIC) that contains 2624 clinical cases, the presented approach achieves an accuracy of 93.1\%. Pomponiu et al. [18] presents a method that automatically extracts a set of representative features for diagnosing skin lesions, pre-trained deep neural network (DNN) used for diagnosing skin lesions, knearest -neighbor classifier used for the classification, 399 images apply on the KNN method with the result of $93.64 \%$ accuracy.

In current study the researcher proposed a system to detect and predict the skin tumour by using case-based reasoning method and expert system and also present the similarity rate between similar cases and their features which is useful for the medical field for detecting skin tumor in the early stage and safe human life through this, and to prevent the painful derma test for the patients.

\section{SKIN CANCER}

Skin is considered as the largest part of the human body [1]. Cancer is considered as a common issue in the medical field. Cancer starts when the cells are uncontrolled growth [2]. Skin cancer is the most common type of cancer. This type of cancer happens in humans with the fair-skin and commonly in the type 1 and type 2 of skin color according to the Fitzpatrick scale who exposed to the sun and other x-radiation and ultraviolet light for a long period of time [19]. There are three types of skin cancer which are the basal cell carcinoma, Squamous cell carcinoma, melanoma [3].

\section{1 basal cell carcinoma $(B C C)$}

$\mathrm{BCC}$ is the type of skin cancer which is a non-melanoma skin cancer (NMSC). BCC arises from the basal layer of the epidermis, and it is the common type of skin cancer found in humans which is approximately (75\% to $80 \%$ ) of all skin cancers [20]. This type of cancer appears mostly in the face and neck, in this type of cancer the Metastasis is extremely rare in case if it is spread it leads to poor functional and cosmetic outcomes [21] figure 1 shows the basal cell carcinoma.

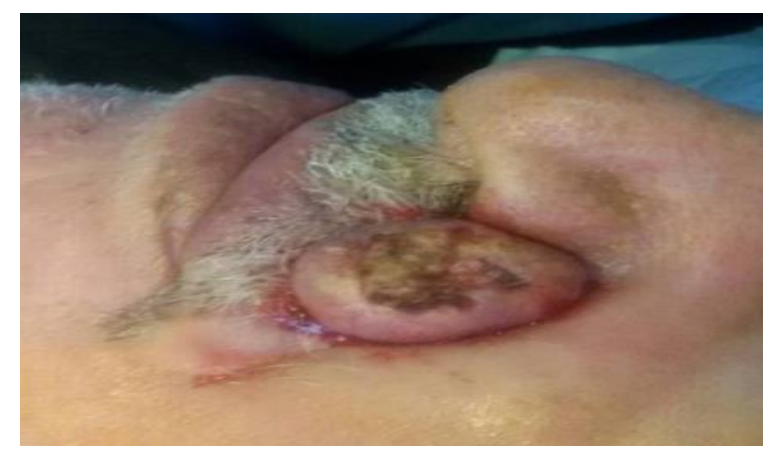

Figure 1: Basal cell carcinoma

\subsection{Squamous cell carcinoma (SCC)}

SCC is the type of non-melanoma skin cancer that is developed from the squamous cell and arises from basal keratinocytes of the skin. Squamous cell carcinoma less occurred than basal cell carcinoma, but it is more spread than BCC and metastasize in $2 \%$ to $5 \%$ of cases and causes another type of cancer (secondary tumor). It mostly occurs in the hand, ear, lip, and another part of the body that is exposed to the sun and tobacco use and the parts of the body 
that have UV exposure [22] [3] [23]. Figure 2, shows Squamous cell carcinoma in the right check and the SCC metastasizes and developed a secondary tumor.

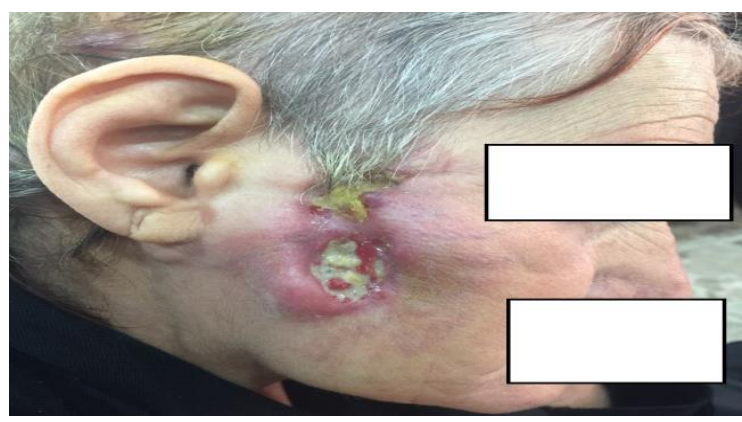

Figure 2: Shows Squamous cell carcinoma and the SCC metastasize and developed a secondary tumour.

\subsection{Melanoma skin cancer:}

Malignant melanoma is the type of skin cancer which is known as melanoma skin cancer (MSC). It is the less common cancer that occurs in humans compared to the BCC and SCC. Melanoma skin cancer happens as a bleeding mole or changing the mole size and shape and color. This type of cancer is easily spread and it is a serious form and needs to be created early because later it is difficult to be treated [24]. Figure 3, present the melanoma skin cancer.

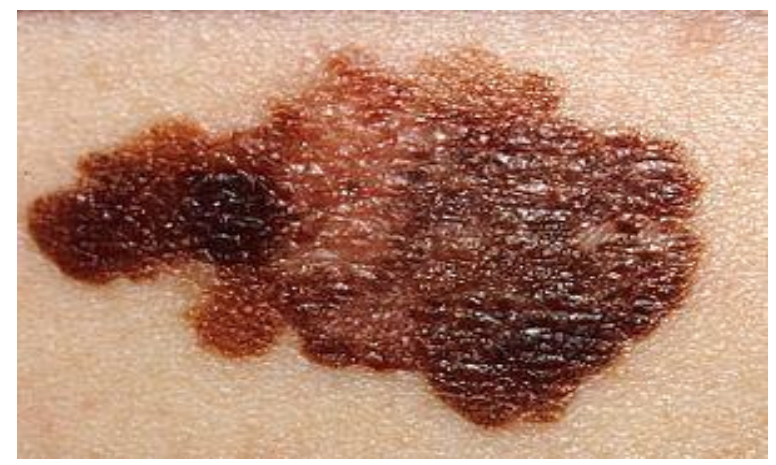

Figure 3: Melanoma skin cancer [2]

\section{ARCHITECTURE DESIGN}

The architecture design for the proposed system includes the case-based reasoning (CBR) method and the expert system. The proposed system is used for predicting skin tumor and shows the similarity rate between the target image and the source image. In the CBR method, when a new case detects as a target image, the image pre-processed and analyzed by using image processing technique, and also re-analyzed by the expert doctor, and extract features from the image, and the image stored in the knowledge base (KB) [25]. Then the image starts with the case-based reasoning cycle which begins with the RETRIEVE step which is responsible for retrieving a similar case, the target problem retrieve from memory cases to solve the problem [26], after that the REUSE step begins, in this step the solution of the target problem or the solution of the most similar case reused for the new cases. [27] when the new case suggested then in the REVISED step the suggested solution tested to know if it is possible to use, this step is used to make the evaluation of the applicability of the solution that suggested from the reused step .The RETAIN step is the last step for the CBR method, in this step the tested solution added to the database and retains the learned base to be used in the future [28]. Figure 4, shows the case-based reasoning cycle. 


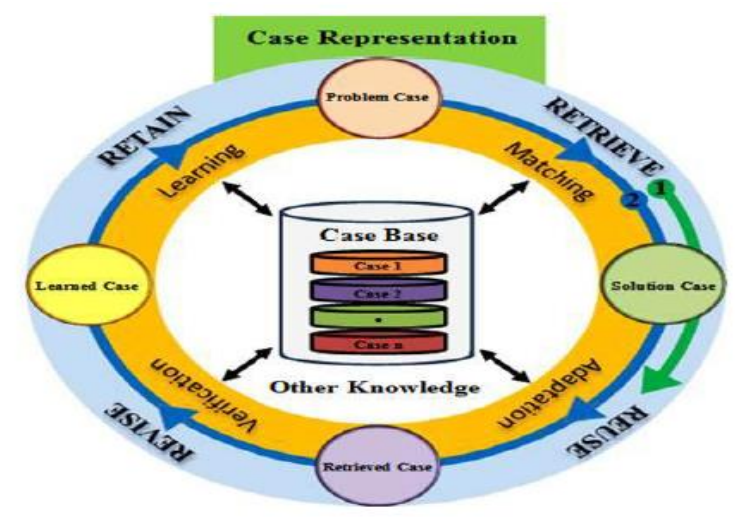

Figure 4: Case-Based Reasoning (CBR) Cycle [27]

\section{PROPOSED SYSTEM}

The proposed system for skin cancer detection consists of two phases, the first phase is adding archival cases and the second phase is testing the new case. the proposed system compares the skin lesion image of a new case (target image) with the old cases (source image) in the knowledge base (KB), each case represented with its own weight and information related to the skin lesion, and the proposed system find the most similar case to the target case, and reuse the solution of the similar case for the target case, after that the solution tested for the new case to represent if it is possible to use or not, and in the last step the evaluation for the new case or the learned case store in the database to be one of the source cases and it is ready to be re-use for the future use. Figure 5, presents the proposed system work. 


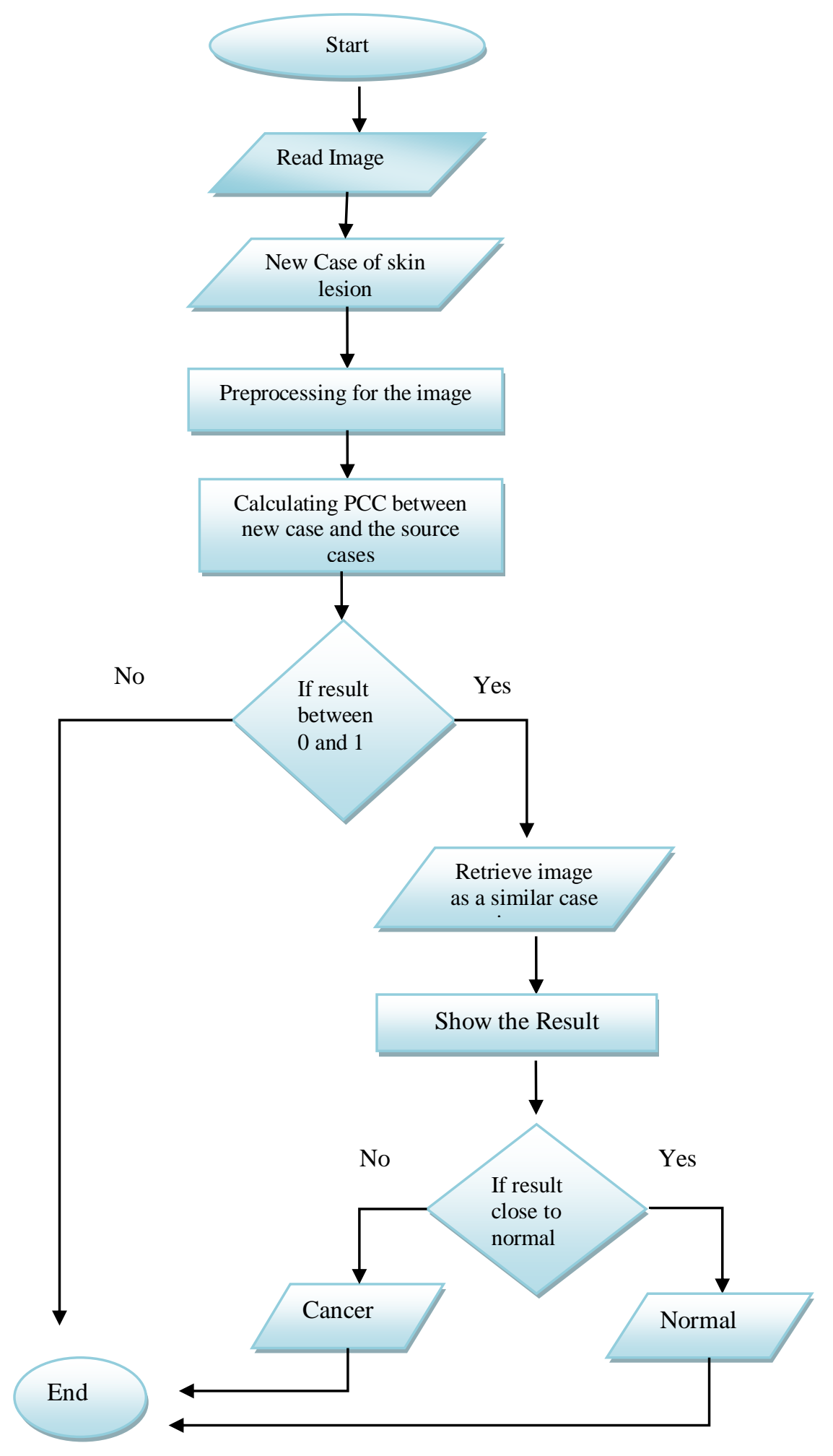

Figure 5: flow chart of the proposed system work. 


\section{1 phases of the proposed system:}

The first phase of the proposed system for skin cancer detection is adding archival cases to form our knowledge base. Each new case includes a patient skin lesion image and information about the skin lesion ( by filling the questionnaire)by the expert doctor, then pre-processing done on the image after that, both image and digitized information put in the database (knowledge Base).figure 6, present the first phase of the proposed system.

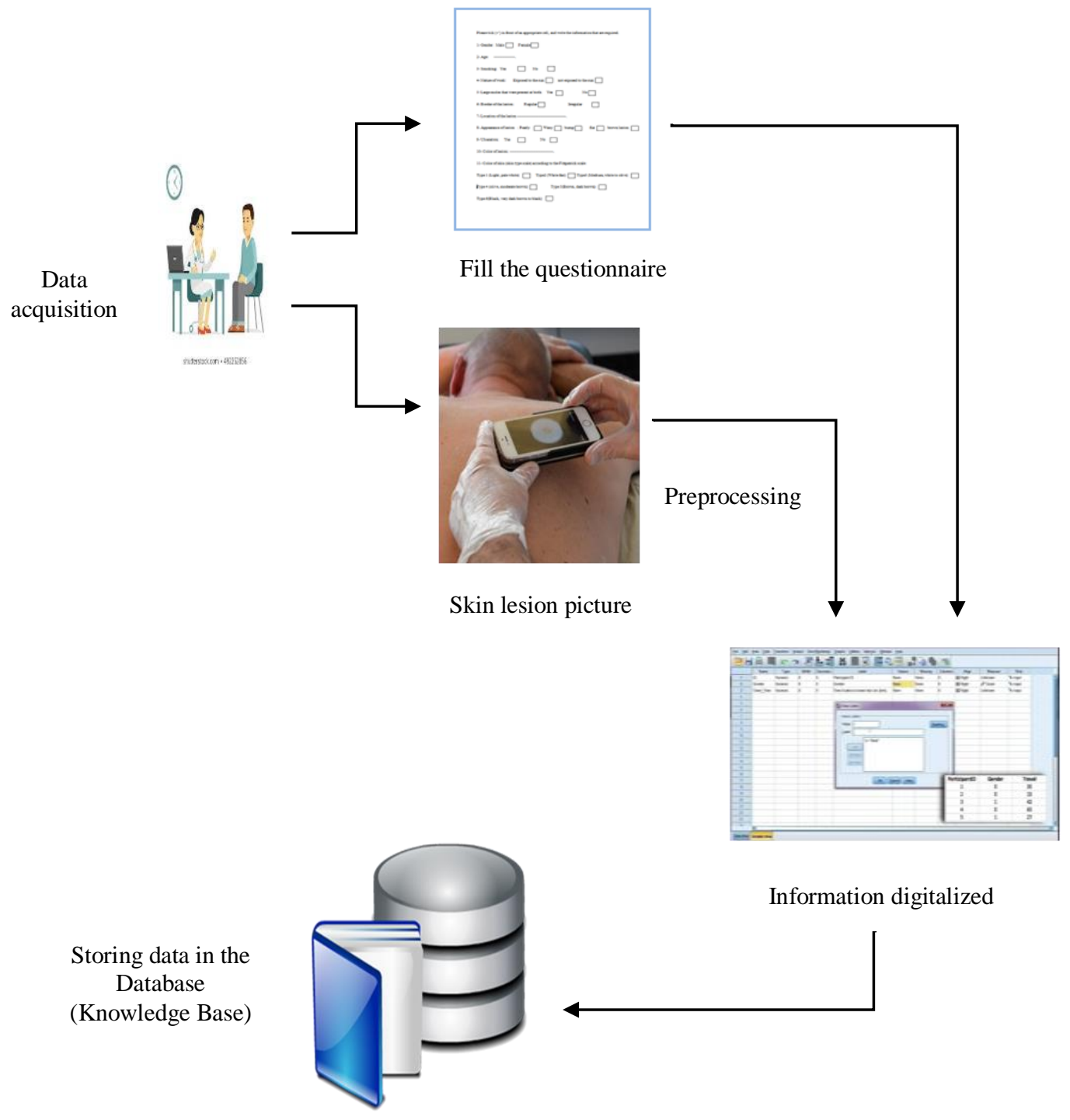

Figure 6: first phase (archiving data) of the proposed system 
In the second phase of the proposed system, the archived image, and information of the target case compared with the images and information in the source cases by using the (PCC) function, the result of the similar cases sent to the case-based reasoning to select the most similar cases and solve the image problem by testing the similar image and find the most similar case to the target case, if it is suitable then select the case as a new learned case. Figure 7 represents the second phase of the proposed system.

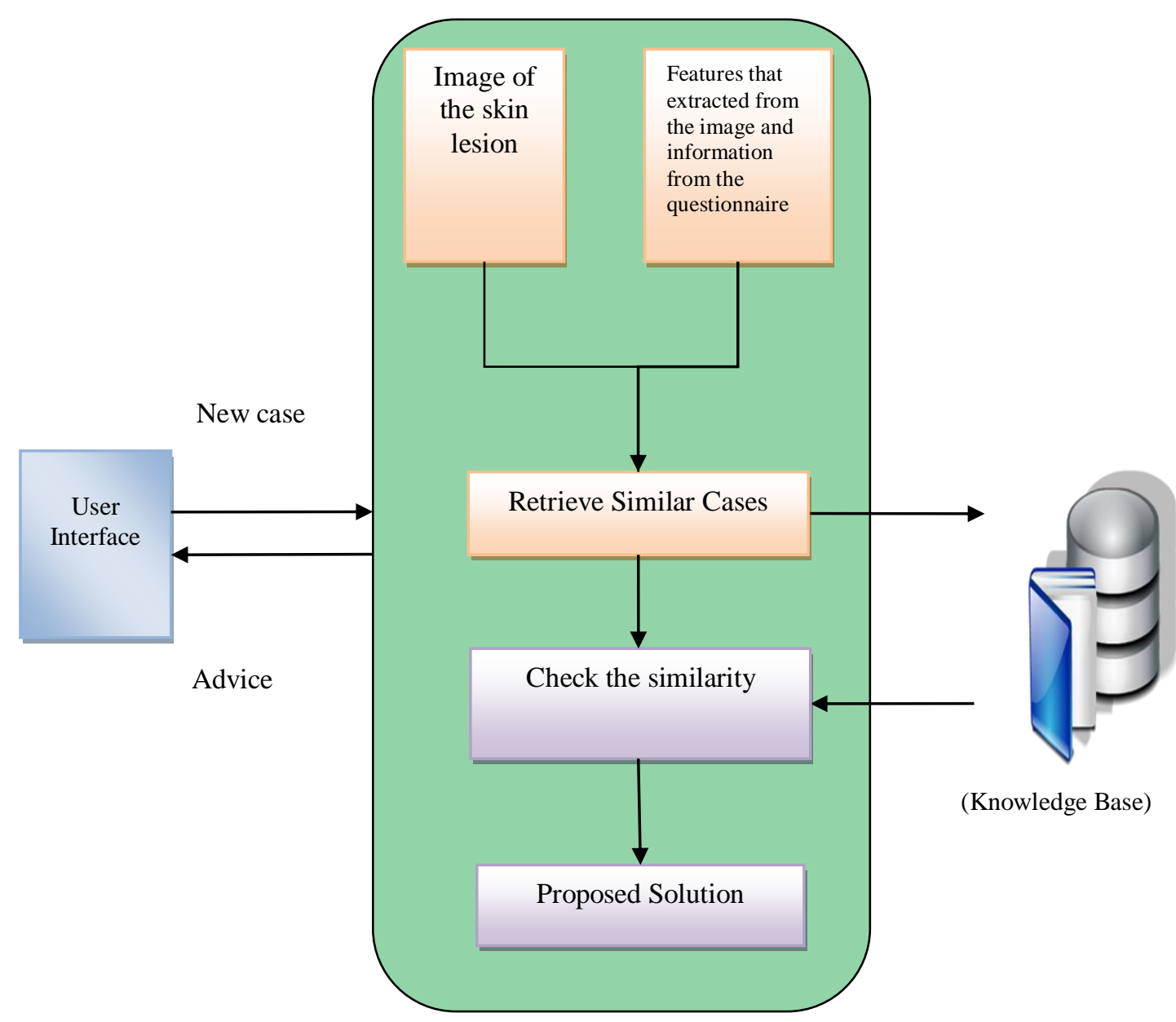

Figure 7: second phase of the proposed system 


\section{DATA COLLECTION AND INVESTIGATIONS}

\subsection{Datasets:}

Three different data sets are used and they are:

Emergency Hospital Burn Surgery Center:

44 Cases have been collected and they are composed of 8 normal and the rest of cancer cases, they are diagnosed by our case expert. Images all are 1280 x 960 pixels.

Hiwa hospital:

15 Cases were collected from hiwa hospital all of the cases are biopsy based proved cancer cases, the report of the cases was diagnosed by the shar hospital lab, histopathology department and as the second decisions all cases are re diagnosed by the specialized expert doctor, The images are all of a good quality, images was taken by using the digital camera the size of images are different some of images are 640x480 pixels and another's are 960x1280 pixels.

Ali kamal hospital:

50 Cases collected from the Ali kamal hospital which consists of 22 normal cases and 28 cancer cases, images taken by the digital camera, size of the images are different.

Xabat skin center:

14 Cases collected, three of them are normal cases and the rest are cancer cases, images taken from digital camera with 960x1280 pixels.

Private clinics:

22 Cases collected, six cases are normal cases and the rest are cancer cases, images taken from digital camera with 960x1280 pixels.

Standard dataset from the DermIS:

206 cases collected from the DermIS [29], 119 of the cases are melanoma, and the rest of non melanoma skin cancer; all images are 550x538 pixels.

\section{EXPERT SYSTEM}

The case-based reasoning system for detecting skin cancer firstly needs the information from the expert (doctor) in the same field which is the derma doctor and the plastic surgery doctor. After the data collection and diagnosing then the feature extracted from the image and data to detect the cancer lesion, after that the all data converted to the information and encoded to the proposed system, and the processed data and the learned case in the knowledge base (KB) for the future use. Figure 8, show the expert system. 


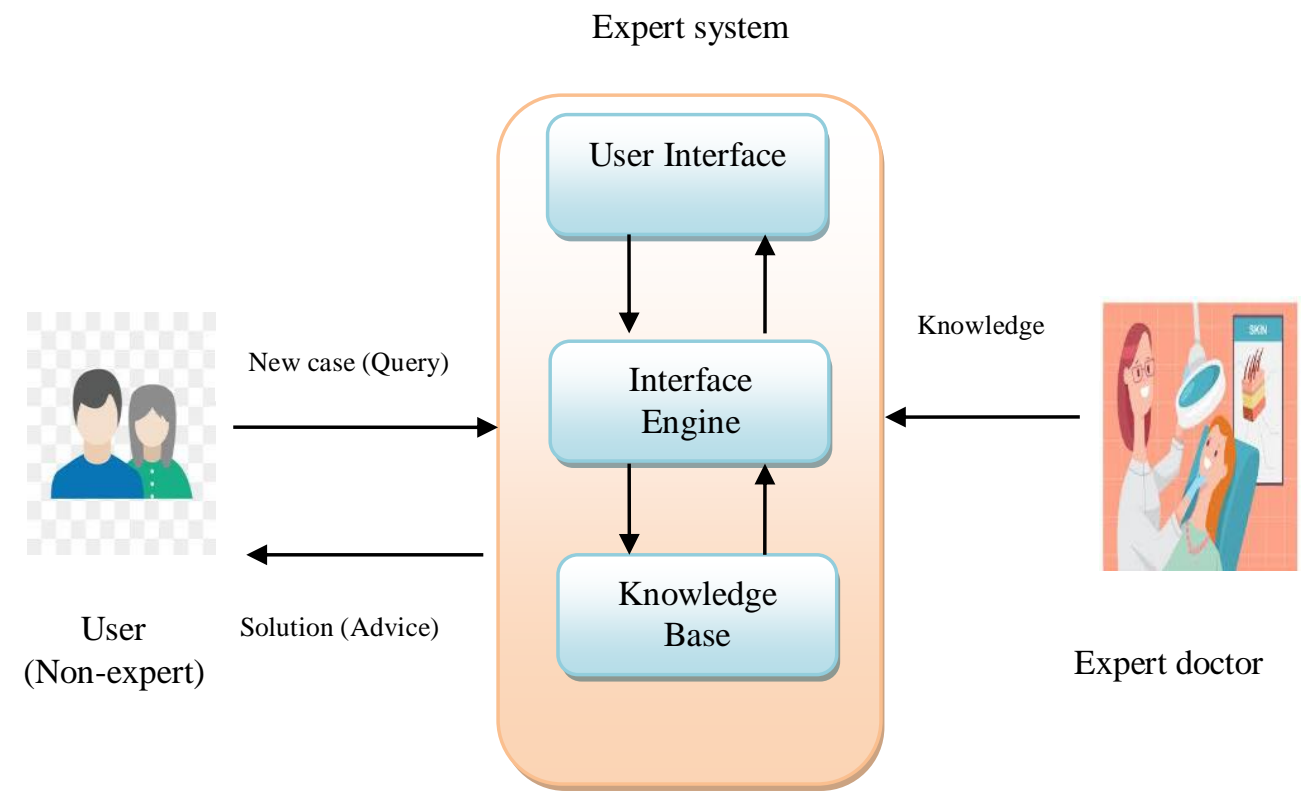

Figure 8: the main Components of an Expert System

\section{REPRESENTATION OF THE CASES}

When new cases enter the system, the case includes the questionnaire and the image of the skin lesion. Each case is about the skin lesion, represented in the knowledge base as the following features of the skin lesion:

ID: to represent the id of the patient and used as an index in the CBR system.

- $\quad$ Age: $(10-20,21-30,31-40,41-50,51-60,61-70,71-80$, above 80).

- $\quad$ Smoking: (yes, no).

- Nature of work :( exposed to the sun, not exposed to the sun).

- $\quad$ Border of the lesion: (regular, irregular).

- Color of skin (skin scale): (type I, type II, type III, type IV, type V, type VI) according to the Fitzpatrick scale [30].

- Diameter: (greater than $1 / 4 \mathrm{~cm}$, less than $1 / 4 \mathrm{~cm}$ ).

- Duration of the lesion: (Less than 12 months, More than 12 months, Unknown).

- The experience of the experts is very important to detect the well-known feature in the skin cancer weight such as, nature of work, Duration of the lesion and other features. In the case-based reasoning system, the index is important for the retrieval step, in the proposed system the ID of the patient and the image required as an index. The experts experience is the most important feature in prediction of skin cancer is the nature of work, then the border of the lesion, after that the duration of the lesion and then other features. 


\section{IMAGE PRE PROCESSING}

The proposed system needs the image and the information for the similarity process. First the image of the skin lesion captured then the image cropped in order to show the region on interested area (ROI) [31] all images are with size $128 * 128$ pixels [7] figure 7, shows the image and the cropped image (ROI) image.

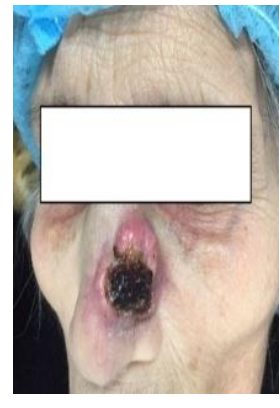

a. The whole image

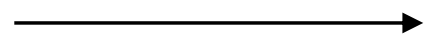

Figure 9: Shows the image and the cropped image (ROI) image.

\subsection{Wavelet Transform:}

Image-processing includes varying operations such as resizing the image, copying, displaying and so on. Unfortunately, these manipulations lead to degrading the image quality and made many kinds of noise. In the proposed system, noise removal achieved by using the mathematical function called wavelet function. Wavelet used for image compression and denoising image.

When wavelet applied on the image the wavelet image consist of the low-frequency sub-band which describes the global image (LL) part and The approximation of sub-band symbol as LL and the detail sub-bands symbol as LH, HL, and $\mathrm{HH}[32]$. The compression of an image works as shown in the figure 8.

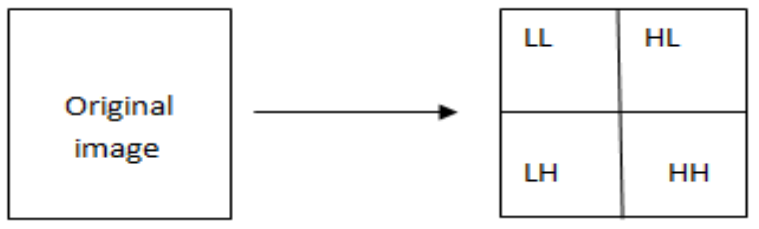

Figure 10: wavelet transforms compression of an image.

In the figure 11 presents the wavelet function applied on the image by using a small program that written with Visual Studio C\# 2015 to remove noise from the image. 


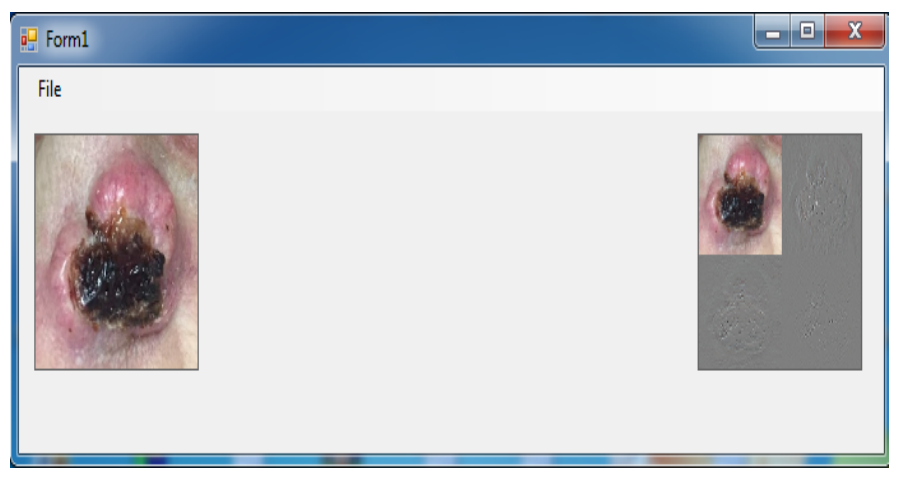

Figure 11: wavelet applied on the image

After this step the LL part takes from the image, and this leads to resize the image, figure 12 show this step.

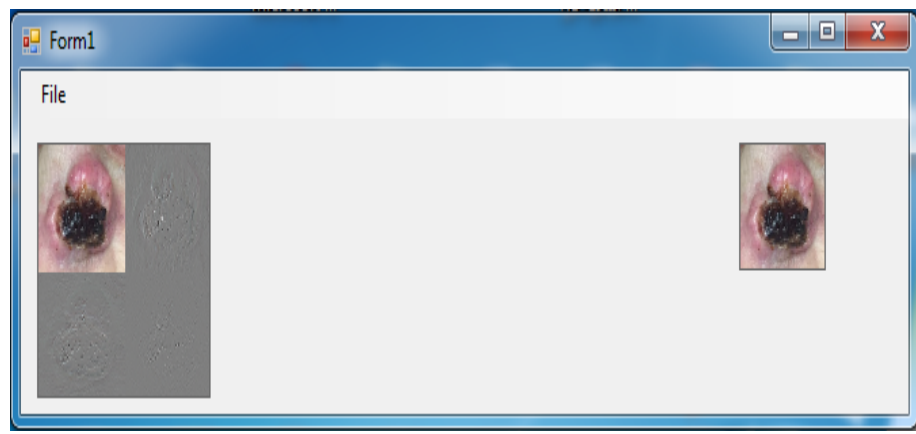

Figure 12: Extract the LL part from the image

\section{2 gray scale:}

Converting a color image into the grayscale image is one of the image processing techniques. The grayscale image is also called the achromatic image. The contrast shade range of the grayscale is between 0 which is the black color to 255 which is the white color; the rest of the color between 0 and 255 is the grey color [33].in the proposed system the image that wavelet function apply on it and the LL take from the image converted into the grayscale image as show in the figure 13.

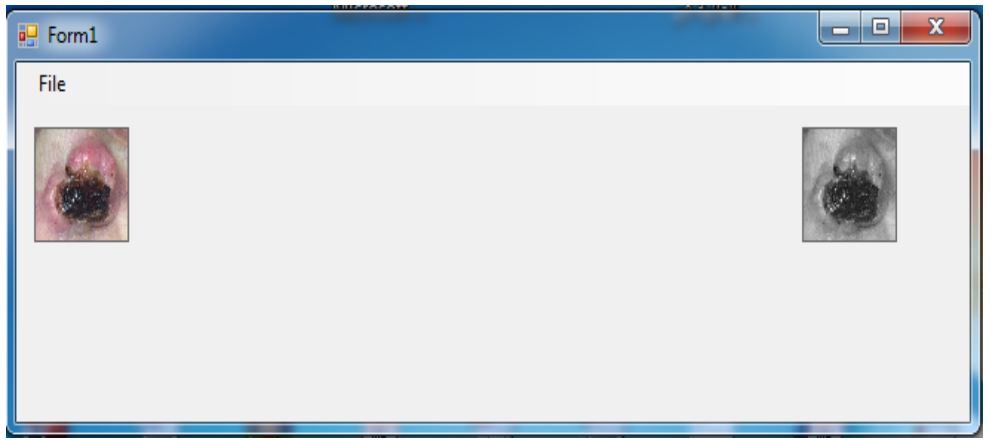

Figure 13: The RGB image converted to the grey scale 


\section{SIMILARITY FOR THE IMAGE}

The pre processed image which is the target image entered to the proposed system to check the similarity rate between the target image and the source image which are the cancer table and normal table from the database which is built by using Microsoft access, the similarity rate stored in the table in the database, and the proposed system choose the nearest result which is the similar case to the target by using the PCC (equation (1)) [34].

$$
r_{1}=\frac{\sum_{i}\left(x_{i}-x_{m}\right)\left(y_{i}-y_{m}\right)}{\sqrt{\sum_{i}\left(x_{i}-x_{m}\right)^{2}} \sqrt{\sum_{i}\left(y_{i}-y_{m}\right)^{2}}}
$$

$\mathrm{Xi}, \mathrm{Yi}$ : represents the intensity value for the target case.

$\mathrm{Xm}, \mathrm{Ym}$ : represent the intensity value of the source image in the proposed system.

The proposed system checks the pixel by pixel's image's similarity, and finds the relation and similarity rate between images. The images similarity rate filled with $(0$ and 1$)$, if the result is so then there is the similarity between images but if the result is negative skin means that there is no connection between images, and the proposed system neglect the negative result. Depending on the result the proposed system retrieves the similar case from the knowledge base and tests the case and store the target case in to a database as a learned case to be ready for the future use.

\section{IMPLEMENTATION AND RESULT}

For the proposed system to predict and define the skin cancer works as below:

After the pre-processing of the image, the pre-processed image and data for the patient entered into the system for analyzing data and image. in the proposed system all data converted to numbers for easy calculation by using statistics method by weighting parameters, after entering the data the target image loaded into the system to compare the target image and target data to the source image and source data in the knowledge base (KB), then the system shows the similar cases based on the number of cases that the expert wanted to see. The result of the similar cases shows in the table with their own images, after that the system adds the target data into a table in the source table and also adds the target image into the source image to be a learned case and to be ready for the future use. Figure 14 shows the process of the comparison and presentation of the similar cases in the system. 


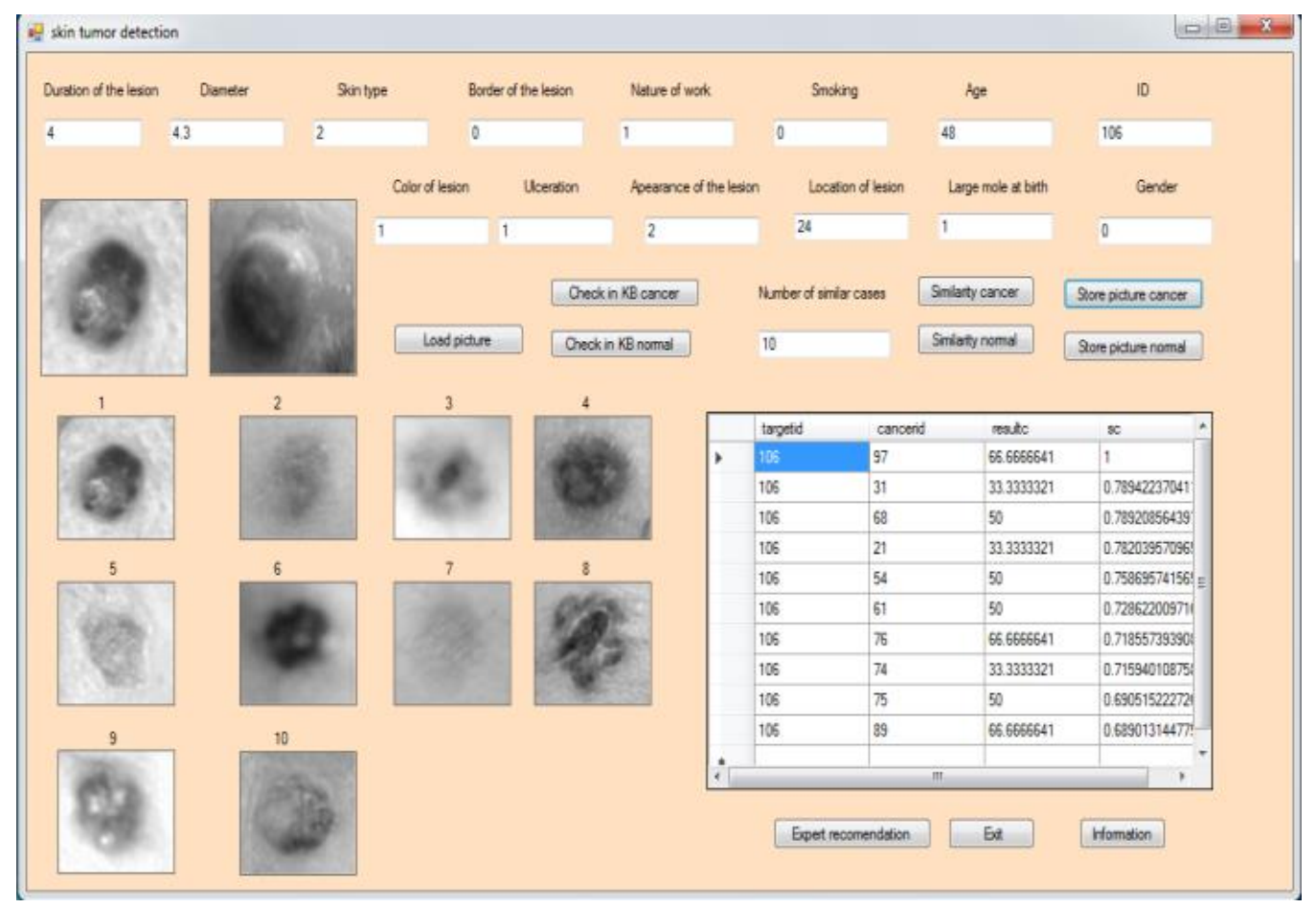

Figure 14: The proposed system's work

In the interface of the system that showed in the previous figure shows that the first step is entering data for the patient or new case or the target case. Figure 15 shows the data entry.

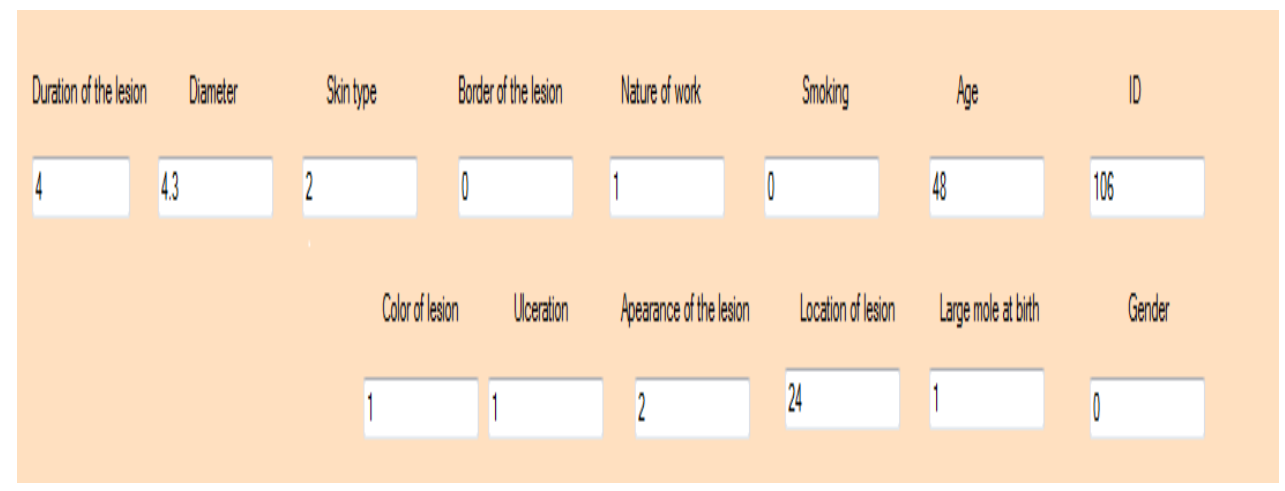

Figure 15: shows the data entry

After the data entry then by clicking on the load picture button, the target image loaded in to the system, then by click on the check in KB cancer the process of comparing between the target image and the source image (cancer) in the knowledge base, and the same process done for the normal case by clicking on the check in $\mathrm{KB}$ normal .In figure 16 , shows the comparison process. 


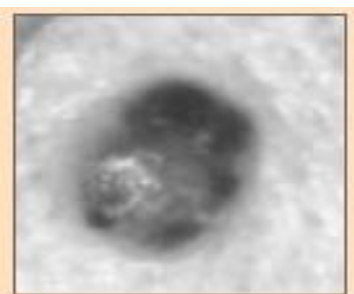

A

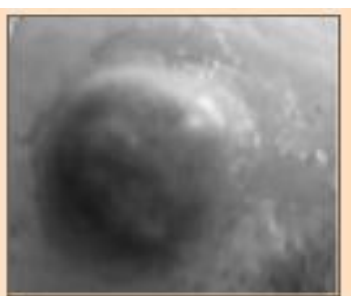

B

Figure 16: Comparing process

A. represents the target image.

B.represent the source image which is changes the image over the time, for example if there are 350 cases in the source, so the target image compare with all 350 cases in the source. When the comparing process done then the process of presenting similarity begins with clicking on the similarity cancer button for presenting the similar cancer cases, and the same process for the normal cases by clicking on the similarity normal, the number of the similar cases entered by the expert manually for example in the figure 17, shows the 10 similar cases with their data.

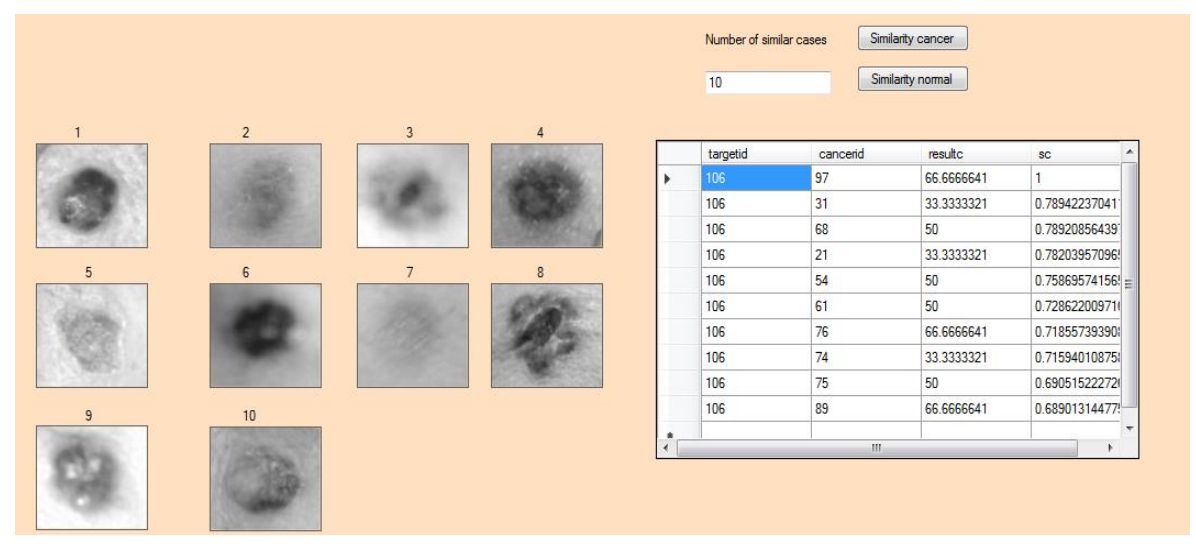

Figure 17: shows the similar cases with their data

When the similar cases presented then the target case stored as a new learned case in the cancer source by click on the store picture cancer, and if you want to store the case in the normal case you can click on the store picture normal as a new learned case to be ready for the future use. 


\section{BENCH MARK}

Through analyzing the methods and algorithms that are used for diagnosing and detecting skin tumors from the previous papers, these results achieved which is shown in table 1.This table contains the algorithms and methods that used for skin tumor detection, and some of their advantages and drawbacks. The proposed system worked on the diagnosing skin tumor by using the image processing tools, case based reasoning and expert system, PCC function used for measuring the similarity between images with the accuracy from $68 \%$ to $100 \%$.

Table 1: presentation of the benchmarks

\begin{tabular}{|c|c|c|c|}
\hline Paper/author(s) & Method/algorithm & $\begin{array}{c}\text { Accurac } \\
\mathbf{y}\end{array}$ & $\begin{array}{l}\text { Reference } \\
\text { s }\end{array}$ \\
\hline $\begin{array}{l}\text { V. Pomponiu, H. Nejati and N. Cheung, } \\
\text { "Deepmole: Deep neural networks for skin } \\
\text { mole lesion classification," } 2016 \text { IEEE } \\
\text { International Conference on Image } \\
\text { Processing (ICIP), Phoenix, AZ, pp. 2623- } \\
\text { 26272016. }\end{array}$ & $\begin{array}{l}\text { k-nearest neighbors } \\
(\mathrm{KNN})\end{array}$ & $93.64 \%$ & [18] \\
\hline $\begin{array}{l}\text { N. Codella, J. Cai, M. Abedini, R. Garnavi, } \\
\text { A. Halpern and J. R. Smith," Deep Learning, } \\
\text { Sparse Coding, and SVM for Melanoma } \\
\text { Recognition in Dermoscopy Images," } \\
\text { 10.1007/978-3-319-24888-2,2015 }\end{array}$ & SVM & $93.1 \%$ & [17] \\
\hline $\begin{array}{l}\text { S. Kolkur,D.R. Kalbande, V. Kharkar," } \\
\text { Machine Learning Approaches to Multi- } \\
\text { Class Human Skin Disease Detection," } \\
\text { International Journal of Computational } \\
\text { Intelligence Research ,Vol. 14, No. } 1 \text {,pp. 29- } \\
\text { 39, } 2018\end{array}$ & Neural network & $\begin{array}{c}90 \% \\
\text { above }\end{array}$ & {$[10]$} \\
\hline $\begin{array}{l}\text { Vijayalakshmi M M,” Melanoma Skin } \\
\text { Cancer Detection using Image Processing } \\
\text { and Machine Learning," International } \\
\text { Journal of Trend in Scientific Research and } \\
\text { Development (IJTSRD),vol. 3,no. 4,pp.780- } \\
\mathbf{7 8 4 , 2 0 1 9}\end{array}$ & $\begin{array}{l}\text { Convolutional Neural } \\
\text { Network and Support } \\
\text { Vector Machine and } \\
\text { amalgamated it with } \\
\text { image processing tools }\end{array}$ & $85 \%$ & [16] \\
\hline $\begin{array}{l}\text { J. Kawahara, A. BenTaieb, and G. } \\
\text { Hamarneh," Deep Features to Classify Skin } \\
\text { Lesions," 1397-1400. } \\
\text { 10.1109/ISBI.2016.7493528,2016. }\end{array}$ & linear classifier & $81.8 \%$ & [15] \\
\hline
\end{tabular}

\section{CONCLUSION}

This paper, described different types of skin cancer and different techniques for the target image's preprocessing (image of the skin lesion) such as, cropping the image to detect the (ROI) area, applying the wavelet transform function to reduce the noise from the image and to compress the image, and converting the color image into the grayscale image. The case-based reasoning and expert system used to detect and make a prediction for skin cancer. after the pre-processing the pre-processed image entered into the CBR system to retrieve the image and reuse the image to solve the problem and test it if it is solved then revise the image to confirm the solution and retain the image as a learned case and stored in the knowledge base for the future use. For the case-based reasoning, it needs the expert doctor to diagnose the case and extract features from the case and data encoded to use in the proposed system to detect skin cancer. 


\section{REFERENCE}

[1] H. Yousef and S. Sharma, " Anatomy, Skin (Integument)," Epidermi., 2017.

[2] S. R. Silp and V. chidvila, "A Reveiw On Skin Cancer," international research journal of pharmacy, vol. 4, no. 8, pp. 1-8, 2013.

[3] M. I. Qadir, "Skin Cancer: Etiology And Management," Pakistan journal of pharmaceutical sciences, vol. 29, no. 3, pp. 1-6, 2016.

[4] Sh. Jaina, V. jagtap and N. Pise, "Computer Aided Melanoma Skin Cancer Detection Using Image," International Conference on Intelligent Computing, Communication \& Convergence(ICCC),pp. 1-6, 2015.

[5] M. Chandrahasa, V. Vadigeri and D.Salecha, "Detection Of Skin Cancer Using Image Processing Techniques," International Journal of Modern Trends in Engineering and Research(IJMTER), vol. 3, no. 5, pp. 111-114, 2016.

[6] H. C.Fernández,O.L.Ortega and F. Castro-Espinozaa, V. Ponomaryov, "An Intelligent System For The Diagnosis Of Skin Cancer On Digital Images Taken With Dermoscopy," Acta Polytechnica Hungarica, vol. 14, no. 3, pp. $169-185,2017$

[7] R. P. Periyasamy and V.Gayathiri, "Melanoma Detection Through K-Means Segmentation And Feature Extraction," International Research Journal of Engineering and Technology (IRJET), vol. 4, no. 5, pp. 1301$1305,2017$.

[8] M. Anas, R. K. Gupta and Dr. Sh. Ahmad, "Skin Cancer Classification Using K-Means Clustering," International Journal of Technical Research and Applications, vol. 5, no. 1, pp. 62-65, 2017.

[9] A.M. Khirala, A. Sibai, Y .A. Abdarrahim and M. A. Omer, "A Literature Study Of Wavelets And Their Applications," 10.13140/RG.2.2.22856.32008,2017.

[10] S. Kolkur, D.R. Kalbande and V. Kharkar, "Machine Learning Approaches to Multi-Class Human Skin Disease Detection," International Journal of Computational Intelligence Research, vol. 14, no. 1, pp. 1-12, 2018.

[11] M. A. M. Shukran, N. M. S. Ahmad, S. Ramli and F. Rahmat, "Melanoma Cancer Diagnosis Device Using Image Processing Techniques," International Journal of Recent Technology and Engineering (IJRTE), vol. 7, no. 5S7, pp. 490-494, 2019.

[12] S. Bindhu and M. Mohan, "Segmentation Of Skin Lesions Using Texture Distinctiveness Lesion Segmentation Algorithm," Algorithm, vol. 1, no. 2, pp. 56-60, 2015.

[13] S.Kannan, V. Gurusamy and G.Nalini,"Review On Image Segmentation Techniques,” 2014.

[14] J.B. Lamy, B. Sekarb, G. Guezenneca, J. Bouauda and B.Séroussia, "Explainable artificial intelligence for breast cancer: A visual case-based reasoning approach," Artificial Intelligence In Medicine, 94. 10.1016/j.artmed.2019.01.001, 2019.

[15] J. Kawahara, A. Ben Taieb, and G. Hamarneh," Deep Features to Classify Skin Lesions," 1397-1400. 10.1109/ISBI.2016.7493528,2016.

[16] Vijayalakshmi M M," Melanoma Skin Cancer Detection using Image Processing and Machine Learning," International Journal of Trend in Scientific Research and Development (IJTSRD),vol. 3,no. 4,pp.780-784,2019.

[17] N. Codella, J. Cai, M. Abedini, R. Garnavi, A. Halpern and J. R. Smith,” Deep Learning, Sparse Coding, and SVM for Melanoma Recognition in Dermoscopy Images," 10.1007/978-3-319-24888-2,2015.

[18] V. Pomponiu, H. Nejati and N. Cheung, "Deep mole: Deep neural networks for skin mole lesion classification," 2016 IEEE International Conference on Image Processing (ICIP), Phoenix, AZ, pp. 2623 26272016.

[19] S. Sachdeva, "Fitzpatrick skin typing: Applications in dermatology," Indian J Dermatol Venereol Leprol, vol. 75, no. 1, pp. 93-96, 2009.

[20] S. Chung, "Basal Cell Carcinoma," Continuing Medical Education, vol. 39, no. 2, pp. 166-170, 2012.

[21] P. P. de Freitas, C. G. Senna,M. Tabai,C. T. Chone and A. Altemani, "Metastatic Basal Cell Carcinoma: A Rare Manifestation of a Common Disease," Hindawi Case Reports in Medicine, vol. 2017, pp. 1-5, 2017.

[22] N. DurgaRao and G.Sudhavani, "A Survey on Skin Cancer Detection System," Journal of Engineering Research and Application, vol. 7, no. 6, pp. 59-64, 2017.

[23] W. Yan, I. I. Wistuba, M. R. Emmert-Buck and H. S. Erickson, "Review Article Squamous cell carcinoma similarities and differences among anatomical sites," Am J Cancer Res, vol. 1, no. 3, pp. 1-26, 2011.

[24] P. Das, N.Deshmukh, N. Badore, C. Ghulaxe and P. Patel, "A Review Article on Melanoma," journal of pharmaceutical sciences and research, vol. 8, no. 2, pp. 112-117, 2016.

[25] S. Gupta and R. Singhal, "Fundamentals and Characteristics of an Expert System," International Journal on Recent and Innovation Trends in Computing and Communication, vol. 6, no. 3, pp. 110-114, 2013.

[26] S. V. Shokouhi, p .Skalle and A.Aamodt, "An overview of case-based reasoning applications in drilling engineering," Artificial Intelligence Review, 41. 10.1007/s10462-011-9310-2, 2014.

[27] M. A. Mohammed, B. Al-Khateeb and D. A.Ibrahim, "Case based Reasoning Shell Frameworkas Decision Support Tool," Indian Journal of Science and Technology, vol. 9, no. 42, pp. 1-8, 2016.

[28] H. Y. A. Abutair, A. Belghith, "Using Case-Based Reasoning for Phishing Detection ," Procedia Computer Science, 109. 281-288. 10.1016/j.procs.2017.05.352,2017.

[29] Melanoma images, DermIS http://www.dermis.net

[30] S. Sachdeva," Fitzpatrick skin typing: Applications in dermatology," Indian journal of dermatology, venereology and leprology. 75. 93-6. 10.4103/0378-6323.45238,2009.

[31] A. Wasilewska, J. Pauk and M. I. Touski," Image Processing Techniques For Roi Identification In Rheumatoid Arthritis Patients From Thermal Images," Acta Mechanica et Automatica, vol. 12, no. 1,pp. 49-53,2018.

[32] S. Ross-Howe and H.R. Tizhoosh ," The Effects of Image Pre- and Post-Processing, Wavelet Decomposition, and Local Binary Patterns on U-Nets for Skin Lesion Segmentation" Accepted for publication in proceedings of the IEEE World Congress on Computational Intelligence (IEEE WCCI), Rio de Janeiro, Brazil, 8-3 July, 2018. 
[33] C. Saravanan, “Color Image to Grayscale Image Conversion,” 196 - 199. 10.1109/ICCEA.2010.192,2010.

[34] A. Kaur, L. Kaur and S. Gupta," Image Recognition using Coefficient of Correlation and Structural Similarity Index in Uncontrolled Environment," International Journal of Computer Applications, vol. 59, no. 5, pp. 32 39,2012 . 